\title{
Robust Stability Clearance of Flight Control Law Based on Global Sensitivity Analysis
}

\author{
Liuli Ou, Lei Liu, Shuai Dong, and Yongji Wang \\ Key Lab of Ministry of Education for Image Processing \& Intelligent Control, School of Automation, \\ Huazhong University of Science and Technology, 1037 Luoyu Road, Wuhan 430074, China
}

Correspondence should be addressed to Lei Liu; liulei@hust.edu.cn

Received 16 June 2014; Revised 23 August 2014; Accepted 23 August 2014; Published 13 October 2014

Academic Editor: Weichao Sun

Copyright (C) 2014 Liuli Ou et al. This is an open access article distributed under the Creative Commons Attribution License, which permits unrestricted use, distribution, and reproduction in any medium, provided the original work is properly cited.

To validate the robust stability of the flight control system of hypersonic flight vehicle, which suffers from a large number of parametrical uncertainties, a new clearance framework based on structural singular value $(\mu)$ theory and global uncertainty sensitivity analysis (SA) is proposed. In this framework, SA serves as the preprocess of uncertain model to be analysed to help engineers to determine which uncertainties affect the stability of the closed loop system more slightly. By ignoring these unimportant uncertainties, the calculation of $\mu$ can be simplified. Instead of analysing the effect of uncertainties on $\mu$ which involves solving optimal problems repeatedly, a simpler stability analysis function which represents the effect of uncertainties on closed loop poles is proposed. Based on this stability analysis function, Sobol's method, the most widely used global SA method, is extended and applied to the new clearance framework due to its suitability for system with strong nonlinearity and input factors varying in large interval, as well as input factors subjecting to random distributions. In this method, the sensitive indices can be estimated via Monte Carlo simulation conveniently. An example is given to illustrate the efficiency of the proposed method.

\section{Introduction}

In the past decades, clearance of flight control system has been paid great attention by the air force of many countries. Rational clearance improves not only the reliability and safety of flight control system, but also feedback valuable information to designers. Clearance methods split neatly into two types based on the different clearance principle. One is the so-called analytical-model-based method (AMBM) [1] and another is simulation-data-based method (SDBM) $[2,3]$. AMBM analyses stable margin, robust stability and some other criteria based on advanced control theories which include (1) structural singular value $(\mu)$ analysis $[4,5]$; (2) polynomial-based clearance [6]; (3) v-gap analysis; (4) bifurcation and continuation method [7]; and (5) optimizationbased clearance [8]. AMBM has been improved and extended to more criteria and more types of air vehicles [9-14]. SDBM demands high fidelity simulation platform and combines experiment design techniques with decision sciences. Generally speaking, AMBM is suitable for early stages of the whole controller designing process, whereas SDBM is suitable for late stages. These two types of methods are complementary.
For hypersonic flight vehicle (HFV) whose maximum flight speed may reach to about 20 Mach, the principal criterion to validate is robust stability of its attitude control system because of the existence of uncertain coefficients. The uncertainties are mainly caused by the following three factors. First, the aerodynamic coefficients of HFV, obtained via wind tunnel test and computational fluid mechanics tools, often suffer from much more serious deviations than those of supersonic and subsonic vehicles. Second, it is still unknown how the external environment affects the HFV due to lack of flight experience. Finally, erosion and corrosion may damage the aerodynamic configuration of the vehicle and hence change the aerodynamic coefficients. Many advanced methods [15-17] have the potential to improve the ability and performance of the attitude control system, which may also lead to more complicated controllers and require more rational clearance.

Among these model-based clearance methods mentioned above, $\mu$ analysis is the favourable method for robust stability criterion due to its nonconservatism. Calculation of $\mu$ with pure real uncertainties [18] had always been a challenge until the optimization-based approach [19] was proposed. Hybrid 


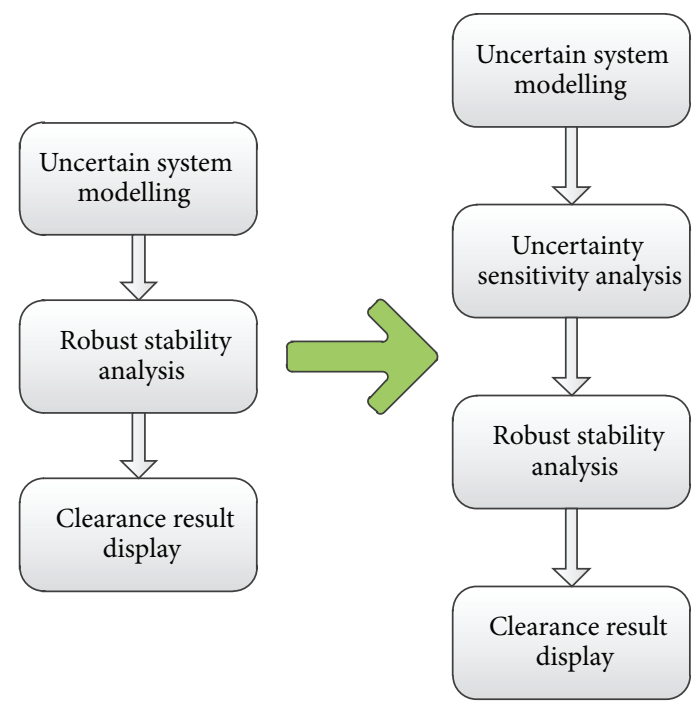

FIgURE 1: The new clearance process.

optimization [8], which combines sequential quadratic programming (SQP) with intelligent optimization, can produce an exact estimation of $\mu$. However, the computational burden is still heavy due to iteratively sweeping frequencies. In particular, when the number of uncertainties is big, it will take a long time to get $\mu$ values at a series of frequency points.

In order to reduce the computational burden of $\mu$, engineers usually ignore some unimportant uncertain factors according to their experience. How to find those minor uncertain factors of the HFV when we have little experience? The answer is sensitivity analysis (SA). SA methods can be broken down into local SA [20,21] and global SA [22-25]. Global SA has been applied widely in industrial production, ecological engineering, hydrology systems, and so on [26] due to its two main advantages. First, it is suitable for input factors varying in broad range and system model with strong nonlinearity. Second, it can consider input factors obeying certain random distributions.

In this work, we proposed a new clearance framework for robust stability clearance of the control system of HFV based on $\mu$ theory and global uncertainty SA (GUSA), which is shown in Figure 1. The GUSA serves as the preprocess of the $\mu$ analysis to help engineers to determine those minor uncertainties. Because measuring the direct effect of uncertainties on $\mu$ involves computing $\mu$ repeatedly, a novel indirect GA index based on our newly defined stability analysis function is adopted. This function represents the effect of uncertainties on dominant closed loop poles. With this new GA index, we can extend the widely used Sobol's method [23] and apply it to analyse the effect of uncertainties on stability of the flight control system. It is worth noting that the framework is suitable for not only $\mu$ analysis but also other robust analysis approaches. Furthermore, GUSA can provide valuable information about the effect of aerodynamic coefficients on stability for subsequent design and validation.

The paper is organized as follows. In Section 2, the uncertain model of the attitude control system of HFV is established and $\mu$ framework is presented. The Sobol's method is extended and applied to GUSA in Section 3. Section 4 gives an example to illustrate the efficiency of the proposed method. In Section 5, some conclusions are stated.

\section{Problem Description}

The motion equations of the hypersonic flight vehicle are given as follows [27]:

$$
\begin{gathered}
\dot{\alpha}=\frac{C_{A} q S_{m} \sin \alpha-C_{N} q S_{m} \cos \alpha}{m v \cos \beta}-\frac{\omega_{x} \cos \alpha \sin \beta}{\cos \beta} \\
+\frac{\omega_{y} \sin \alpha \tan \beta+\omega_{z}-g_{y}}{v \cos \beta} \\
\dot{\beta}=\frac{C_{A} q S_{m} \cos \alpha \sin \beta+C_{N} q S_{m} \sin \alpha \sin \beta+C_{z} q S_{m} \cos \beta}{m v} \\
+\frac{g_{z}+\omega_{x} \sin \alpha+\omega_{y} \cos \alpha}{v} \\
\dot{\omega}_{x}=\frac{C_{m x} q S_{m} l_{k}}{J_{x}}+\frac{C_{l p} q S_{m} l_{k}^{2} \omega_{x}}{2 v J_{x}}+\frac{\left(J_{y}-J_{z}\right) \omega_{y} \omega_{z}}{J_{x}} \\
\dot{\omega}_{y}=\frac{C_{m y} q S_{m} l_{k}}{J_{y}}+\frac{C_{n r} q S_{m} l_{k}^{2} \omega_{y}}{2 v J_{y}}+\frac{\left(J_{z}-J_{x}\right) \omega_{z} \omega_{x}}{J_{y}} \\
\dot{\omega}_{z}=\frac{C_{m z} q S_{m} l_{k}}{J_{z}}+\frac{C_{m q} q S_{m} l_{k}^{2} \omega_{z}}{2 v J_{z}}+\frac{\left(J_{x}-J_{y}\right) \omega_{x} \omega_{y}}{J_{z}},
\end{gathered}
$$

where

$$
\begin{aligned}
& \alpha, \beta, \gamma=\text { attack, sideslip, and roll angle; } \\
& \omega_{z}, \omega_{y}, \omega_{x}=\text { pitch, yaw, and roll rate; } \\
& C_{A}, C_{N}, C_{Z}=\text { aerodynamic coefficients; } \\
& C_{m x}, C_{m y}, C_{m z}=\text { aerodynamic moment coefficients; } \\
& C_{l p}, C_{n r}, C_{m q}=\text { damping moment coefficients; } \\
& J_{x}, J_{y}, J_{z}=\text { inertia moment in } x, y, z \text { axes; } \\
& S_{m}, l_{k}=\text { wing area and span; } \\
& q=\text { dynamic pressure; } \\
& m=\text { mass; } \\
& v=\text { velocity; and } \\
& \delta_{\varphi}, \delta_{\psi}, \delta_{\gamma}=\text { elevator, rudder, and aileron deflection. }
\end{aligned}
$$

These aerodynamic coefficients are nonlinear functions of the deflection angles $\delta_{\varphi}, \delta_{\psi}$, and $\delta_{\gamma}$. Consider

$$
C_{i}=f_{i}\left(\delta_{\varphi}, \delta_{\psi}, \delta_{\gamma}\right), \quad i=A, N, Z, m x, m y, m z, l p, n r, m q
$$

whose nominal values are given in the form of look-up tables. $C_{i}, q, J_{x}, J_{y}$, and $J_{z}$ are uncertain coefficients varying in some certain intervals around their nominal values. 


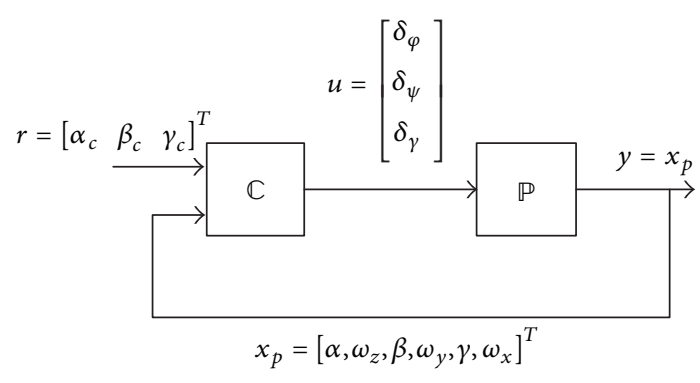

FIGURE 2: Closed loop system of the attitude control of HFV.

Linearizing (1) and (2) along the nominal flight trajectory according to the methods introduced in $[4,18]$, the uncertain model $\mathbb{P}$ can be written as

$$
\dot{x}_{p}=A_{p} x_{p}+B_{p} u
$$

where $x_{p}=\left[\begin{array}{llllll}\alpha & \omega_{z} & \beta & \omega_{y} & \gamma & \omega_{x}\end{array}\right]^{T}$ is the state vector, $u=$ $\left[\begin{array}{lll}\delta_{\varphi} & \delta_{\psi} & \delta_{\gamma}\end{array}\right]^{T}$ is the control input, and $A_{p} \in \mathfrak{R}^{6 \times 6}$ and $B_{p} \in$ $\mathfrak{R}^{6 \times 3}$ are uncertain matrices in the affine form as follows:

$$
\begin{aligned}
& A_{p}=A_{p 0}+\sum_{i=1}^{k} \sigma_{i} A_{p i} \\
& B_{p}=B_{p 0}+\sum_{i=1}^{k} \sigma_{i} B_{p i}
\end{aligned}
$$

where $k$ is the number of normalized uncertainties after combination and linearization and $\sigma_{i} \in[-1,1]$ are uncertain parameters that represent the uncertainties of these coefficients. The uncertain model (4) is not unique via different modelling methods. Because of different designing and manufacturing environment for different vehicles, uncertainties may obey certain random distribution. Consider

$$
\sigma_{i} \sim R
$$

for instance, the normal distribution $\sigma_{i} \sim N\left(\mu, v^{2}\right)$ or the uniform distribution $\sigma_{i} \sim U(a, b)$.

Suppose that a state feedback controller $\mathbb{C}$, which can stabilize the nominal system of (3), is

$$
\begin{aligned}
& \dot{x}_{c}=A_{c} x_{c}+B_{c 1} x_{p}+B_{c 2} r \\
& u=C_{c} x_{c}+D_{c 1} x_{p}+D_{c 2} r
\end{aligned}
$$

where $x_{c} \in \mathfrak{R}^{d \times 1}$ is the state vector of controller, $r=$ $\left[\begin{array}{lll}\alpha_{c} & \beta_{c} & \gamma_{c}\end{array}\right]^{T}$ is the command attitude angles to be tracked, $A_{c} \in \mathfrak{R}^{d \times d}, B_{c 1} \in \mathfrak{R}^{d \times 6}, B_{c 2} \in \mathfrak{R}^{d \times 3}, C_{c} \in \mathfrak{R}^{3 \times d}, D_{c 1} \in \mathfrak{R}^{3 \times 6}$, and $D_{c 2} \in \Re^{3 \times 3}$.

Then, the closed loop system, as shown in Figure 2, can be written as

$$
\left[\begin{array}{c}
\dot{x}_{p} \\
\dot{x}_{c}
\end{array}\right]=\left(A_{0}+\Delta A\right)\left[\begin{array}{c}
x_{p} \\
x_{c}
\end{array}\right]+\left(B_{0}+\Delta B\right) r
$$

where

$$
\begin{aligned}
& A_{0}=\left[\begin{array}{cc}
A_{p 0}+B_{p 0} D_{c 1} & B_{p 0} C_{c} \\
B_{c 1} & A_{c}
\end{array}\right] \\
& B_{0}=\left[\begin{array}{c}
B_{p 0} D_{c 2} \\
B_{c 2}
\end{array}\right] \\
& \Delta A=\sum_{i=1}^{k} \sigma_{i} A_{i}=\sum_{i=1}^{k}\left(\sigma_{i}\left[\begin{array}{cc}
A_{p i}+B_{p i} D_{c 1} & B_{p i} C_{c} \\
\mathbf{0} & \mathbf{0}
\end{array}\right]\right) \\
& \Delta B=\sum_{i=1}^{k} \sigma_{i} B_{i}=\sum_{i=1}^{k}\left(\sigma_{i}\left[\begin{array}{c}
B_{p i} D_{c 2} \\
0
\end{array}\right]\right) .
\end{aligned}
$$

It is clear that the stability of the system (7), suffering from affine interval uncertainties $\sigma_{i}$, is determined by the system matrix $A_{0}+\Delta A$. In $\mu$ framework, the linear fraction representation (LFR) of (7), denoted as $\mathbb{F}(M, \Delta)$, should be modelled first according to the approach presented in $[4,18]$. Consider

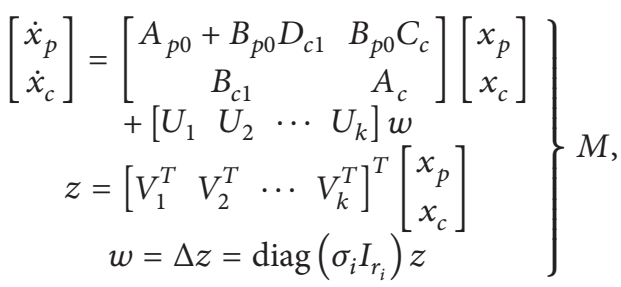

where $M$ represents known part of the system with input $w$ and output $z, \Delta=\operatorname{diag}\left\{\sigma_{i} I_{r_{i}}\right\}$ represents uncertainties of diagonal structure, $\operatorname{rank}(\cdot)$ is the rank of a matrix, and $U_{i} \in$ $\mathfrak{R}^{(n+d) \times r_{i}}$ and $V_{i} \in \mathfrak{R}^{r_{i} \times(n+d)}$ are obtained by full rank decomposition. Consider

$$
\begin{aligned}
& U_{i} V_{i}=\left[\begin{array}{cc}
A_{p i}+B_{p i} D_{c 1} & B_{p i} C_{c} \\
\mathbf{0} & \mathbf{0}
\end{array}\right] \\
& r_{i}=\operatorname{rank}\left(\left[\begin{array}{cc}
A_{p i}+B_{p i} D_{c 1} & B_{p i} C_{c} \\
\mathbf{0} & \mathbf{0}
\end{array}\right]\right) .
\end{aligned}
$$

At a specific frequency point $\omega$, structural singular value of $\mathbb{F}(M, \Delta)(9)$ is defined as

$$
\mu_{\Delta}(M(j \omega))=\frac{1}{\min \left\{\|\Delta\|_{\infty} \mid \operatorname{det}(I-M(j \omega) \Delta)=0\right\}}>0,
$$

where $\|\cdot\|_{\infty}$ is the infinite norm of a matrix. Define $\mu^{*}=$ $\max _{\omega} \mu_{\Delta}(M(j \omega))$. Since $\|\Delta\|_{\infty} \leq 1$, the sufficient condition of robust stability is $\max \left\{\|\Delta\|_{\infty}\right\}<1 / \mu^{*}$; that is, $\mu^{*}<1$. Furthermore, the system stays stable even if the uncertain intervals extend to $\sigma_{i} \in\left[-1 / \mu^{*}, 1 / \mu^{*}\right]$. For simplicity, we denote $\mu_{\Delta}(M(j \omega))$ as $\mu$ hereinafter.

According to definition (11), if the problem

$$
\begin{array}{ll}
\min & \bar{\gamma} \\
\text { s.t. } & (1)\|\Delta\|_{\infty} \leq \bar{\gamma} \\
& (2) \operatorname{det}(I-M(j \omega) \Delta)=0
\end{array}
$$


has an optimal solution $\bar{\gamma}^{*}$, then the estimation of $\mu$ is $1 / \bar{\gamma}^{*}$. By combining global intelligent algorithm (such as particle swarm optimization (PSO) and differential evolution (DE) [28]) with local SQP, this problem can be iteratively solved [8]. In order to get the estimation of $\mu^{*}=\max _{\omega} \mu$, we need to solve the optimal problem (12) at a series of frequency points.

When the number of uncertainties is large, the dimension of the searching space is also large. In order to cover the whole space uniformly, the number of particles of PSO (or analogy of other intelligent algorithms) should exponentially increase with respect to the number of uncertainties. Then, the computational burden will increase exponentially. Therefore, our aim is to find those $\sigma_{i}$ s which affect the stability dramatically and ignore remaining unimportant $\sigma_{i}$ s when calculating $\mu^{*}$. If we measure the effect of uncertainties on $\mu^{*}$ directly, we need to calculate $\mu^{*}$ repeatedly. So we introduce an indirect stability analysis function.

As we know, a system is stable if and only if all of its closed loop poles lie in the left half of the complex plan. Therefore, we define the nonlinear stability analysis function as follows:

$$
f\left(\sigma_{i}\right)=\max _{j}\left\{\operatorname{Re}\left[\lambda_{j}\left(A_{0}+\Delta A\right)\right]\right\},
$$

where $\lambda_{j}(\cdot)$ is the $j$ th eigenvalue of a matrix and $\operatorname{Re}[\cdot]$ is the real component of a complex. The effect of $\sigma_{i}$ on the stability of the closed loop system can be represented by (13) and SA can be invoked. It is clear that the stability analysis function (13) is also suitable for other robust stability clearance method.

All local SA methods need to calculate the differential or derivative; that is, they are based on the analytical model. However, the nonlinear function (13) does not satisfy this requirement. So we will use the global Sobol's method to deal with this problem in the upcoming sections.

\section{Global Uncertainty Sensitivity Analysis}

3.1. Sobol's Method. Consider the nonlinear model in the form

$$
\begin{aligned}
& f: \mathfrak{R}^{k} \longrightarrow \mathfrak{R} \\
& \mathbf{x} \longrightarrow y=f(\mathbf{x}),
\end{aligned}
$$

where $\mathbf{x}=\left[x_{1}, x_{2}, \ldots, x_{k}\right]$ are $k$ independent input factors and $y$ is the scalar output. If $\mathbf{x}$ is defined over $\Omega=\{\mathbf{x} \mid 0 \leq$ $\left.x_{i} \leq 1, i=1,2, \ldots, k\right\}$, then the function can be decomposed as the following form [23]:

$$
\begin{aligned}
f= & f_{0}+\sum_{i} f_{i}+\sum_{i} \sum_{j>i} f_{i j} \\
& +\sum_{i_{1}} \sum_{i_{2}>i_{1}} \sum_{i_{3}>i_{2}} f_{i_{1} i_{2} i_{3}}+\cdots+f_{12 \cdots k},
\end{aligned}
$$

where $f_{i}=f_{i}\left(x_{i}\right), f_{i j}=f_{i j}\left(x_{i}, x_{j}\right)$, and so on. The uniqueness of (15) is guaranteed by

$$
\int_{0}^{1} f_{i_{1}, i_{2}, \ldots, i_{s}}\left(x_{i_{1}}, x_{i_{2}}, \ldots, x_{i_{s}}\right) d x_{i_{w}}=0,
$$

where $1 \leq i_{1}<i_{2}<\cdots<i_{s} \leq k$ and $i_{w} \in\left\{i_{1}, i_{2}, \ldots, i_{s}\right\}$.
Denote $\mathbf{x}_{i_{1} i_{2} \cdots i_{s}}=\left[x_{i_{1}}, x_{i_{2}}, \ldots, x_{i_{s}}\right], 1 \leq i_{1}<i_{2}<\cdots<i_{s} \leq$ $k$, and $\mathbf{x}_{\sim i_{1} i_{2} \cdots i_{s}}$ as the complement of $\mathbf{x}_{i_{1} i_{2} \cdots i_{s}}$; then we have

$$
\begin{aligned}
& \mathbf{x}_{\sim i}=\left[x_{1}, \ldots, x_{i-1}, x_{i+1}, \ldots, x_{k}\right] \\
& \mathbf{x}_{\sim i j}=\left[x_{1}, \ldots, x_{i-1}, x_{i+1}, \ldots, x_{j-1}, x_{j+1}, \ldots, x_{k}\right]
\end{aligned}
$$

and so on. Define the corresponding domain as $\Omega_{i_{1} i_{2} \ldots i_{s}}$ and $\Omega_{\sim i_{1} i_{2} \cdots i_{s}}$; then we have

$$
\begin{aligned}
& \Omega_{i_{1} i_{2} \cdots i_{s}}=\left\{\mathbf{x}_{i_{1} i_{2} \cdots i_{s}} \mid 0 \leq x_{l} \leq 1, l=i_{1}, i_{2}, \ldots, i_{s}\right\} \\
& \Omega_{\sim i_{1} i_{2} \cdots i_{s}}=\left\{\mathbf{x}_{\sim i_{1} i_{2} \cdots i_{s}} \mid 0 \leq x_{l} \leq 1, l \neq i_{1}, i_{2}, \ldots, i_{s}\right\} .
\end{aligned}
$$

These functions in (15) are obtained by

$$
\begin{aligned}
& f_{0}=\int_{\Omega} f(\mathbf{x}) d \mathbf{x} \\
& f_{i}=\int_{\Omega_{\sim i}} f(\mathbf{x}) d \mathbf{x}_{\sim i}-f_{0} \\
& f_{i j}=\int_{\Omega_{\sim i j}} f(\mathbf{x}) d \mathbf{x}_{\sim i j}-f_{i}-f_{j}-f_{0}
\end{aligned}
$$

and similarly for higher orders, where $\int_{\Omega_{i_{1} \cdots i_{r}}} f(\mathbf{x}) d \mathbf{x}_{i_{1} \cdots i_{r}}=$ $\int_{0}^{1} \int_{0}^{1} \cdots \int_{0}^{1} f(\mathbf{x}) d x_{i_{1}} d x_{i_{2}} \cdots d x_{i_{r}}$.

The variance of $y$ is

$$
V=V(y)=\int_{\Omega} f^{2}(\mathbf{x}) d \mathbf{x}-f_{0}^{2} .
$$

And the partial variance is defined as

$$
V_{i_{1} i_{2}, \ldots, i_{s}}=\int_{\Omega_{i_{1} i_{2} \cdots i_{s}}} f_{i_{1} i_{2} \cdots i_{s}}^{2} d \mathbf{x}_{i_{1} i_{2} \cdots i_{s}} \cdot
$$

Then the decomposition (15) leads to the following decomposition of the variance of $y$

$$
V=\sum_{i=1}^{k} V_{i}+\sum_{1 \leq i<j \leq k} V_{i j}+\cdots+V_{12 \cdots k}
$$

The normalized sensitivity index is defined as

$$
\begin{aligned}
& S_{i}=\frac{V_{i}}{V} \\
& S_{i j}=\frac{V_{i j}}{V} \\
& S_{i_{1} i_{2}, \ldots, i_{s}}=\frac{V_{i_{1} i_{2}, \ldots, i_{s}}}{V},
\end{aligned}
$$

where $S_{i}$ are the so-called first-order sensitivity indices and $S_{i j}$ and $S_{i_{1} i_{2}, \ldots, i_{s}}$ are the second-order and the sth-order indices, respectively. $S_{i_{1} i_{2}, \ldots, i_{s}}$ indicates sensitivity of $y$ to the interaction among $x_{i_{1}}, x_{i_{2}}, \ldots, x_{i_{s}}$. So the total sensitivity indices $S_{T i}$ for the input $x_{i}$ is defined as the sum of all indices relating to 
it. For instance, if $k=3$, we have $S_{T 1}=S_{1}+S_{12}+S_{13}+S_{123}$. It is easy to find that

$$
S_{T i}=1-\frac{V_{\sim i}}{V}
$$

and $0 \leq S_{T i} \leq 1$, where $V_{\sim i}$ is the $(k-1)$ th indices of the remaining $k-1$ factors except the $i$ th one.

Because these global sensitivity indices are based on variance, enough samples are needed. The best and most widely used method is Monte Carlo (MC) simulations. factors

Supposing $\mathbf{X} \in \mathfrak{R}^{N \times k}$ is the matrix of $N$ groups of $k$ input

$$
\mathbf{X}=\left(x_{i j}\right)_{N \times k}=\left[\mathbf{X}_{1}, \mathbf{X}_{2}, \ldots, \mathbf{X}_{k}\right]=\left[\mathbf{x}_{1}^{T}, \mathbf{x}_{2}^{T}, \ldots, \mathbf{x}_{N}^{T}\right]^{T},
$$

where $\mathbf{X}_{i} \in \mathfrak{R}^{N \times 1}$ and $\mathbf{x}_{i} \in \mathfrak{R}^{1 \times k}$. $\mathbf{Y} \in \mathfrak{R}^{N \times 1}$ is the corresponding output

$$
\begin{aligned}
\mathbf{Y} & =f(\mathbf{X})=\left[f\left(\mathbf{x}_{1}\right), f\left(\mathbf{x}_{2}\right), \ldots, f\left(\mathbf{x}_{N}\right)\right]^{T} \\
& =\left[y_{1}, y_{2}, \ldots, y_{N}\right]^{T}
\end{aligned}
$$

and $\mathbf{P}$ and $\mathbf{Q}$ are two samples of $\mathbf{X}$ generated by $\mathrm{MC}$. Then we define that

$$
\mathbf{P}_{\mathbf{Q}}^{(i)}=\left[\begin{array}{lllllll}
\mathbf{P}_{1} & \cdots & \mathbf{P}_{i-1} & \mathbf{Q}_{i} & \mathbf{P}_{i+1} & \cdots & \mathbf{P}_{k}
\end{array}\right]
$$

is the combined matrix with column $i$ from $\mathbf{Q}$ and all other $k-1$ columns from $\mathbf{P}$, where $\mathbf{P}_{i}$ and $\mathbf{Q}_{i}$ are samples of $\mathbf{X}_{i}$. Similarly, we define

$$
\mathbf{Q}_{\mathbf{P}}^{(i)}=\left[\begin{array}{lllllll}
\mathbf{Q}_{1} & \cdots & \mathbf{Q}_{i-1} & \mathbf{P}_{i} & \mathbf{Q}_{i+1} & \cdots & \mathbf{Q}_{k}
\end{array}\right] .
$$

The estimation below can be obtained $[24,25]$ as follows:

$$
\begin{aligned}
& \widehat{f}_{0}=\frac{1}{2 N} \sum_{j=1}^{N}\left[f\left(\mathbf{p}_{j}\right)+f\left(\mathbf{q}_{j}\right)\right] \\
& \widehat{V}=\frac{1}{2 N} \sum_{j=1}^{N}\left[f^{2}\left(\mathbf{p}_{j}\right)+f^{2}\left(\mathbf{q}_{j}\right)\right]-\widehat{f}_{0}^{2} \\
& \widehat{V}_{i}=\frac{1}{N} \sum_{j=1}^{N} f\left(\mathbf{q}_{j}\right) f\left(\left(\mathbf{P}_{\mathbf{Q}}^{(i)}\right)_{j}\right)-\widehat{f}_{0}^{2} \\
& \widehat{V}_{\sim i}=\frac{1}{N} \sum_{j=1}^{N} f\left(\mathbf{q}_{j}\right) f\left(\left(\mathbf{Q}_{\mathbf{P}}^{(i)}\right)_{j}\right)-\widehat{f}_{0}^{2} \\
& \widehat{S}_{i}=\frac{\widehat{V}_{i}}{\widehat{V}}, \quad \widehat{S}_{T i}=1-\frac{\widehat{V}_{\sim i}}{\widehat{V}},
\end{aligned}
$$

where $\mathbf{p}_{j}, \mathbf{q}_{j},\left(\mathbf{P}_{\mathbf{Q}}^{(i)}\right)_{j}$, and $\left(\mathbf{Q}_{\mathbf{P}}^{(i)}\right)_{j}$ are, respectively, the $j$ th row of $\mathbf{P}, \mathbf{Q}, \mathbf{P}_{\mathbf{Q}}^{(i)}$, and $\mathbf{Q}_{\mathbf{P}}^{(i)}$.

3.2. Uncertainties Sensitivity Analysis Based on Sobol's Method. It is worth noting that the input factors of the nonlinear model considered in Sobol's method are limited to $0 \leq x_{i} \leq 1$. However, the normalized uncertainties of the flight control system is $\sigma_{i} \in[-1,1]$. One manner is to renormalize the uncertainties to the interval $[0,1]$. Here we will introduce an easier method.

Considering the nonlinear model (14), if its domain of definition is

$$
\Pi=\left\{\mathbf{x} \mid-0.5 \leq x_{i} \leq 0.5, i=1,2, \ldots, k\right\},
$$

we can get the below theorem.

Theorem 1. The nonlinear model (14) defined over $\Pi$ can be decomposed uniquely to (15) with its items (34) if

$$
\int_{-1 / 2}^{1 / 2} f_{i_{1}, i_{2}, \ldots, i_{s}}\left(x_{i_{1}}, x_{i_{2}}, \ldots, x_{i_{s}}\right) d x_{i_{w}}=0
$$

hold for all $1 \leq i_{1}<i_{2}<\cdots<i_{s} \leq k$ and $i_{w} \in\left\{i_{1}, i_{2}, \ldots, i_{s}\right\}$.

Proof. From (31), we can obtain that the members in (15) are orthogonal. That is, for any

$$
\begin{aligned}
& 1 \leq i_{1}<i_{2}<\cdots<i_{s} \leq k \\
& 1 \leq j_{1}<j_{2}<\cdots<j_{r} \leq k
\end{aligned}
$$

if $\left(i_{1}, i_{2}, \ldots, i_{s}\right) \neq\left(j_{1}, j_{2}, \ldots, j_{r}\right)$, there exists

$$
\int_{\Pi} f_{i_{1} i_{2} \cdots i_{s}} f_{j_{1} j_{2} \cdots j_{r}} d \mathbf{x}=0
$$

Then, we have

$$
\begin{aligned}
& \int_{\Pi} f(\mathbf{x}) d \mathbf{x}=f_{0} \\
& \int_{\Pi_{\sim i}} f(\mathbf{x}) d \mathbf{x}_{\sim i}=f_{i}\left(x_{i}\right)+f_{0} \\
& \int_{\Pi_{\sim i j}} f(\mathbf{x}) d \mathbf{x}_{\sim i j}=f_{i j}\left(x_{i}, x_{j}\right)+f_{i}\left(x_{i}\right)+f_{j}\left(x_{j}\right)+f_{0}
\end{aligned}
$$

and so on for high order terms. Obviously, (34) is similar to (19).

Remark. In fact, Sobol's method can be extend to any nonlinear function defined over

$$
\Gamma=\left\{\mathbf{x} \mid \underline{x} \leq x_{i} \leq \bar{x}, i=1,2, \ldots, k\right\},
$$

where $\bar{x}-\underline{x}=1$.

The uncertain matrix $\Delta A$ of the system matrix in (7) can be rewritten as

$$
\begin{aligned}
\Delta A & =\left[\begin{array}{cc}
\sum_{i} \frac{\sigma_{i}}{2}\left(2 A_{p i}+2 B_{p i} D_{c 1}\right) & \sum_{i} \frac{\sigma_{i}}{2} 2 B_{p i} C_{c} \\
\mathbf{0} & \mathbf{0}
\end{array}\right] \\
& =\sum_{i} 2 \bar{\sigma}_{i}\left[\begin{array}{cc}
A_{p i}+B_{p i} D_{c 1} & B_{p i} C_{c} \\
\mathbf{0} & \mathbf{0}
\end{array}\right],
\end{aligned}
$$


where $\bar{\sigma}_{i}=\sigma_{i} / 2 \in[-0.5,0.5]$ are the normalized uncertainties. It is clear that the uncertain space of $\bar{\sigma}_{i}$ is $\Pi$ defined in (30).

Substituting (7) and (36) into the nonlinear function (13), we can get

$$
\begin{aligned}
f(\overline{\boldsymbol{\sigma}}) & \\
=\max _{j}\left\{\operatorname { R e } \left[\lambda _ { j } \left(A_{0}\right.\right.\right. & \\
& \left.\left.\left.+\sum_{i} \bar{\sigma}_{i}\left[\begin{array}{cc}
2 A_{p i}+2 B_{p i} D_{c 1} & 2 B_{p i} C_{c} \\
\mathbf{0} & \mathbf{0}
\end{array}\right]\right)\right]\right\},
\end{aligned}
$$

where $\overline{\boldsymbol{\sigma}}=\left(\bar{\sigma}_{1}, \bar{\sigma}_{2}, \ldots, \bar{\sigma}_{k}\right), \overline{\boldsymbol{\sigma}} \in \Pi$. The Sobol's method can be applied to analyse the effect of uncertainty terms $\bar{\sigma}_{i}$ on the real part of the closed loop poles. The sample matrices of $\overline{\boldsymbol{\sigma}}$ can be generated randomly according to the distribution of $\sigma$.

Generally, the whole clearance process can be summarized into the following.

Step 1: establish the uncertain model of the attitude control system and normalize the uncertainties to $[-0.5,0.5]$.

Step 2: construct stability analysis function as (37) according to the specific uncertain model.

Step 3: generate the sample matrices $P$ and $Q$ of $N$ groups of uncertainties $\bar{\sigma}$.

Step 4: calculate output samples of the stability analysis functions (37) according to $\mathrm{MC}$ simulations with input samples $P$ and $Q$.

Step 5: calculate the SA index according to (29) and ignore the minor uncertainties with relatively small SA index.

Step 6: calculate the singular structure value according to (12).

\section{Examples}

In this section, a numerical example is given to reveal the effectiveness of the proposed method. Consider the nominal flight trajectory of a HFV depicted as Figure 3, from which 30 operation points for robust stability clearance have been selected. At first, we will illustrate the application of the new clearance process with one single point. Then, we will analyse all these operation points together.

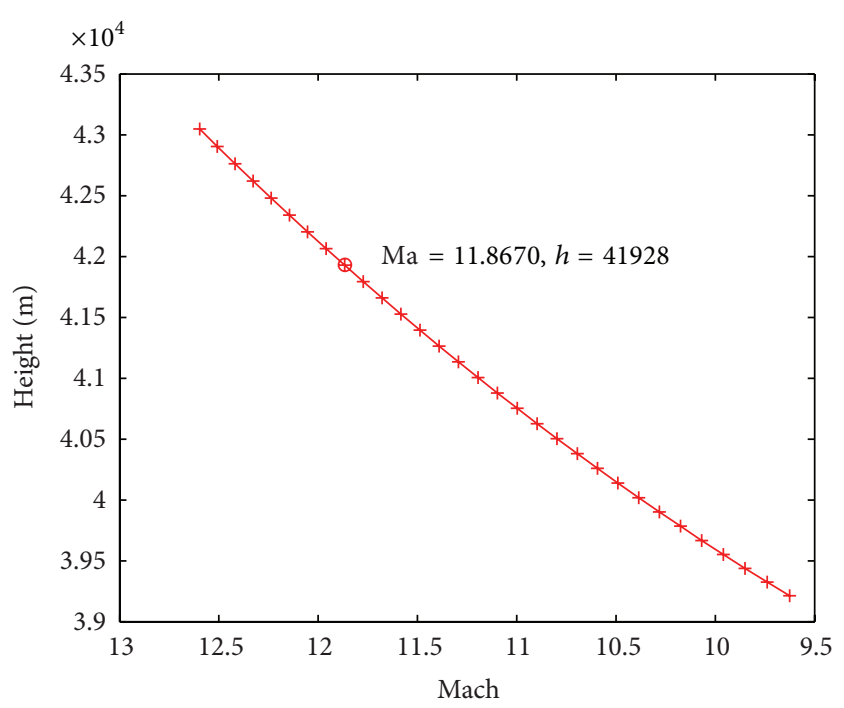

FIgURE 3: Nominal trajectory of the HFV.

At the 9th point $(\mathrm{Ma}=11.8670, h=41928)$, the nominal model of the attitude control equation in the form of (3) is

$$
\begin{gathered}
A_{p 0}=\left[\begin{array}{cc|cccc}
-0.0138 & 1 & 0 & 0 & 0 & 0 \\
-7.6433 & 0 & 0 & 0 & 0 & 0 \\
\hline 0 & 0 & -0.0082 & 0.9725 & 0 & 0.23 \\
0 & 0 & -5.3385 & 0 & 0 & 0 \\
0 & 0 & 0 & 0 & 0 & 1 \\
0 & 0 & -33.8537 & 0 & 0 & 0
\end{array}\right] \\
B_{p 0}=\left[\begin{array}{c|ccc}
-0.0005 & 0 & 0 & \\
-2.5109 & 0 & 0 \\
\hline 0 & -0.0001 & -0.0001 \\
0 & -0.2711 & -1.2441 \\
0 & 0 & 0 \\
0 & -1.1006 & -52.7017
\end{array}\right] .
\end{gathered}
$$

Because the pitch motion has no coupling with yaw and roll motion, the controller can be designed separately. For convenience, a low order controller that can stabilize the nominal system has been designed as follows:

$$
u=\left[\begin{array}{cc}
L_{1} & \mathbf{0} \\
\mathbf{0} & L_{2}
\end{array}\right] r-\left[\begin{array}{cc}
K_{1} & \mathbf{0} \\
\mathbf{0} & K_{2}
\end{array}\right] x_{p}
$$

where

$$
\begin{aligned}
& L_{1}=-99.9845 \\
& L_{2}=\left[\begin{array}{cc}
-3.3664 & 0.6189 \\
-0.2088 & -9.9808
\end{array}\right] \\
& K_{1}=\left[\begin{array}{ll}
-98.1456 & -7.9508
\end{array}\right] \\
& K_{2}=\left[\begin{array}{cccc}
-1.6586 & -3.1971 & 0.6189 & 0.0781 \\
-1.7428 & 2.4028 & -9.9808 & -0.6311
\end{array}\right] .
\end{aligned}
$$

Then the system matrix of the closed loop system is

$$
A=\left[\begin{array}{cc}
A_{c l o 1} & \mathbf{0} \\
\mathbf{0} & A_{c l o 2}
\end{array}\right]+\Delta A,
$$


where

$$
\begin{aligned}
& A_{c l o 1}=\left[\begin{array}{cc}
-0.0587 & 0.9964 \\
-254.0734 & -19.9634
\end{array}\right] \\
& A_{\text {clo } 2}=\left[\begin{array}{cccc}
-0.0085 & 0.9726 & -0.0012 & 0.2329 \\
-7.9562 & 2.1227 & -12.2491 & -0.7640 \\
0 & 0 & 0 & 1 \\
-127.5277 & 123.1129 & -525.3240 & -33.1741
\end{array}\right] \\
& \Delta A=\left[\begin{array}{cccccc}
0 & 0 & 0 & 0 & 0 & 0 \\
\Delta a_{21} & \Delta a_{22} & 0 & 0 & 0 & 0 \\
0 & 0 & 0 & 0 & 0 & 0 \\
0 & 0 & \Delta a_{43} & \Delta a_{44} & \Delta a_{45} & \Delta a_{46} \\
0 & 0 & 0 & 0 & 0 & 0 \\
0 & 0 & \Delta a_{63} & \Delta a_{64} & \Delta a_{65} & \Delta a_{66}
\end{array}\right] \\
& \Delta a_{21}=-1.5287 \sigma_{1}+49.286 \sigma_{4} \\
& \Delta a_{22}=3.9927 \sigma_{4} \\
& \Delta a_{43}=-2.6692 \sigma_{2}+0.0899 \sigma_{5}+0.4336 \sigma_{6} \\
& \Delta a_{44}=0.1733 \sigma_{5}-0.5979 \sigma_{6} \\
& \Delta a_{45}=-0.0336 \sigma_{5}+2.4834 \sigma_{6} \\
& \Delta a_{46}=-0.0042 \sigma_{5}+0.1570 \sigma_{6} \\
& \Delta a_{63}=-6.7707 \sigma_{3}+1.4604 \sigma_{7}+64.2940 \sigma_{8} \\
& \Delta a_{64}=2.8150 \sigma_{7}-88.6422 \sigma_{8} \\
& \Delta a_{65}=-0.5449 \sigma_{7}+368.2036 \sigma_{8} \\
& \Delta a_{66}=-0.0688 \sigma_{7}+23.2820 \sigma_{8} \\
& \sigma_{i} \sim U(-1,1), \quad i=1,2, \ldots, 8 .
\end{aligned}
$$

Calculate the global sensitivity indices of the function (37) with respect to $\bar{\sigma}_{i}=\sigma_{i} / 2 \sim U(-0.5,0.5)$ according to the steps presented in Section 3. In order to illustrate the consistency and repeatability of the proposed method, 10 MC simulations have been conducted. The obtained global sensitivity indices $S_{T i}$ are depicted in Figures 4 and 5. It is evident that (1) the results of 10 simulations are nearly consistent; (2) $S_{T 1}, S_{T 3}$, and $S_{T 4}$ are extremely close to 0 and obviously smaller than other indices; and (3) when the sample number $N$ increases from 20000 to 50000 , the results converge accordingly. Varying $\sigma_{i}$ in the interval $[-1,1]$ singly and fixing $\sigma_{j}=0, j \neq i$, the distribution of the dominant closed loop poles is shown as Figure 6. This can directly validate the SA results.

Since $S_{T i} \approx 0, i=1,3,4$, we can ignore $\sigma_{i}, i=1,3,4$ in the analysis of robust criterion. $\mu$ is calculated via hybrid optimization method at 136 frequency points $\omega=$ $[0.01,0.02, \ldots, 0.09,0.1,0.2, \ldots, 3,4,5, \ldots, 100]$ in the MATLAB environment. Contrastive results are shown in Figures 7 and 8 . When only considering the remaining 5 major uncertainties $\left(\sigma_{i}, i=2,5,6,7,8\right)$, the $\mu$ curve is very close to

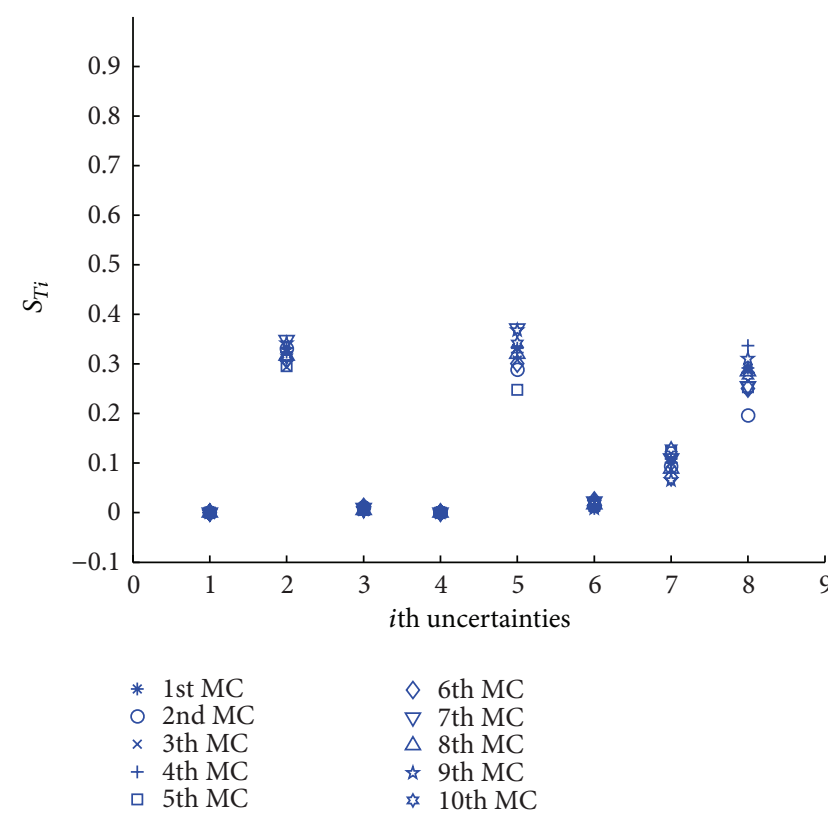

FIGURE 4: The global uncertainty sensitivity indices $(N=20000)$.

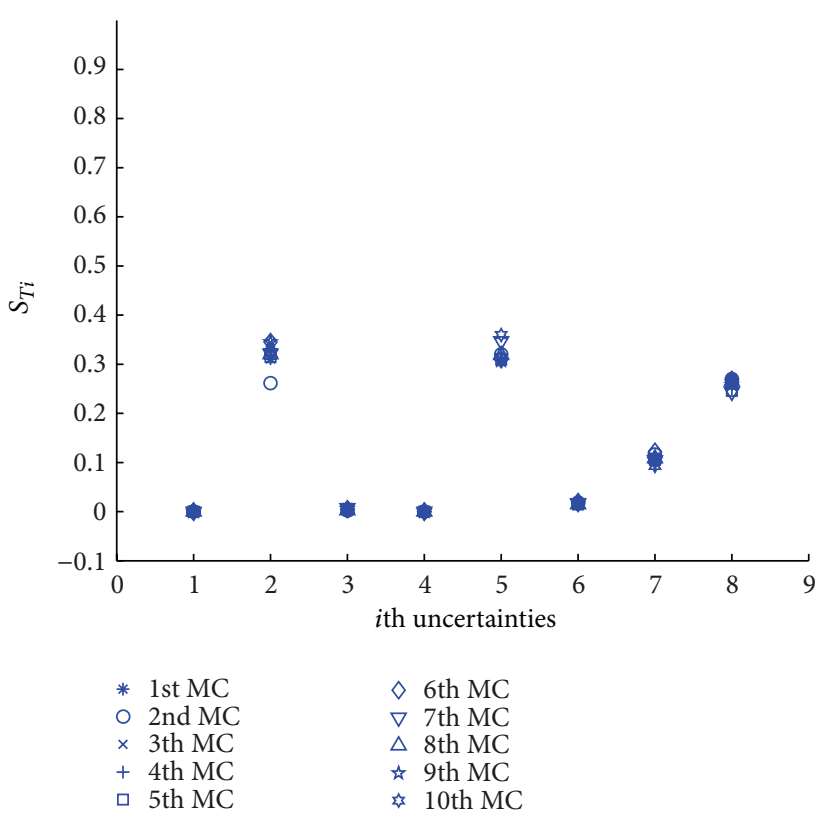

FIGURE 5: The global uncertainty sensitivity indices $(N=50000)$.

the situation of considering all 8 uncertainties. The computational time, however, decreases from $3.3387 \mathrm{~s}$ to $2.2903 \mathrm{~s}$ when the number of uncertainties decreases from 8 to 5 . When taking into account all the operation points together, the proposed clearance frame can save a large amount of time. Because $\mu^{*} \approx 1.1>1$, the controller cannot stabilize the system in the whole uncertain space. However, we can get the valuable information that the approximate allowable uncertain space is $\left|\sigma_{i}\right|<1 / 1.1 \approx 0.9$ and feed it back to designers.

The global sensitivity indices $S_{T i}$ of all operation points are plotted in Figure 9. Note that $S_{T i} \approx 0, i=1,3,4$, holds for 

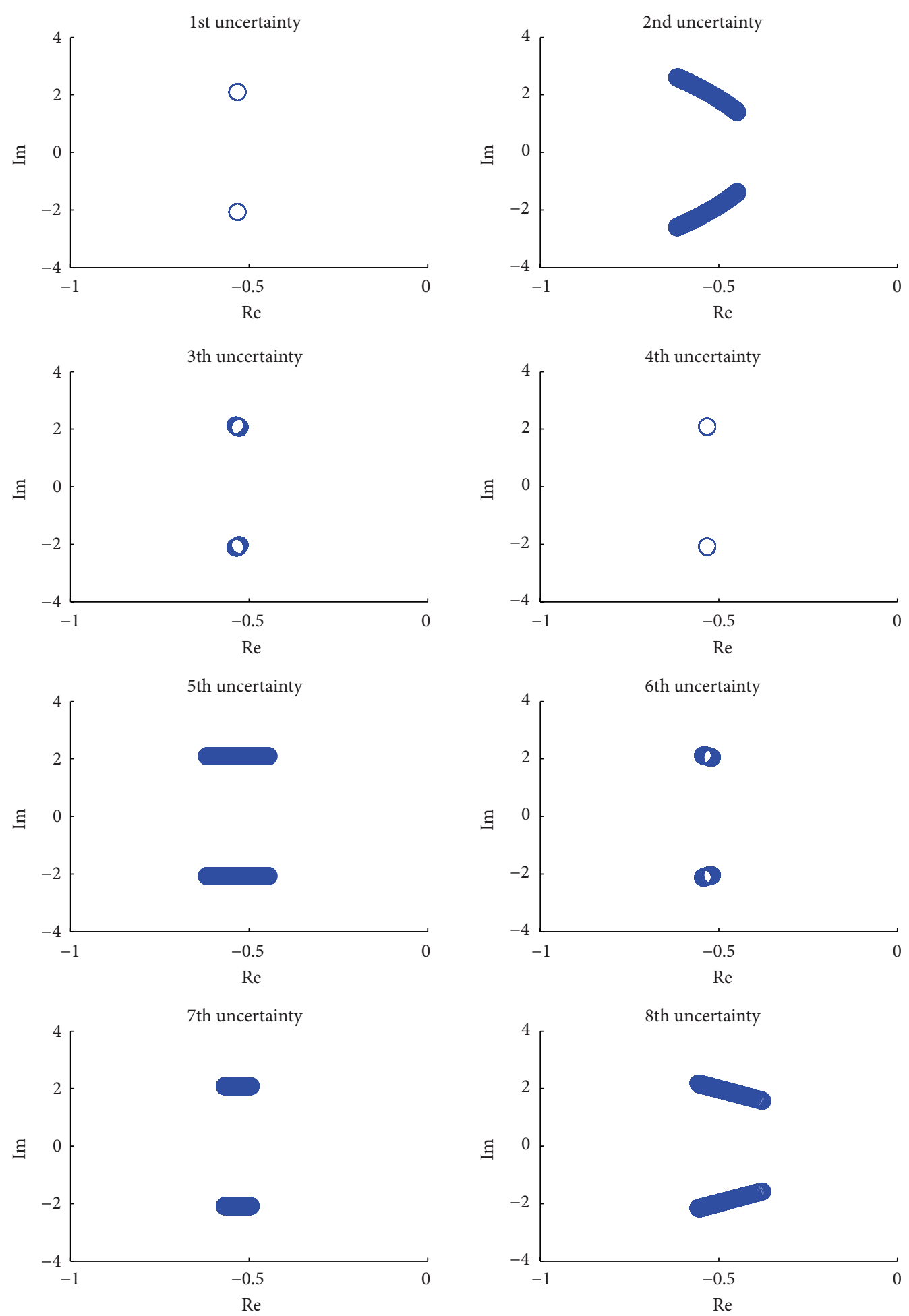

FIgURE 6: Dominant poles distributions for systems with one uncertainty.

all operation points. That is, because the flight trajectory in Figure 3 is relatively smooth and the uncertain model varies slowly. Therefore, we just need to analyse a few operation points when the trajectory is smooth. A comparison of computational time of $\mu$ is also depicted in Figure 10. With the proposed clearance framework, we can save more than 30 s totally while the GUSA just takes 3.6645 s.

In summary, GUSA provides a quantitative index for engineers to measure the effect of uncertainties on dominant closed loop poles. It is worth noting that the GUSA index 


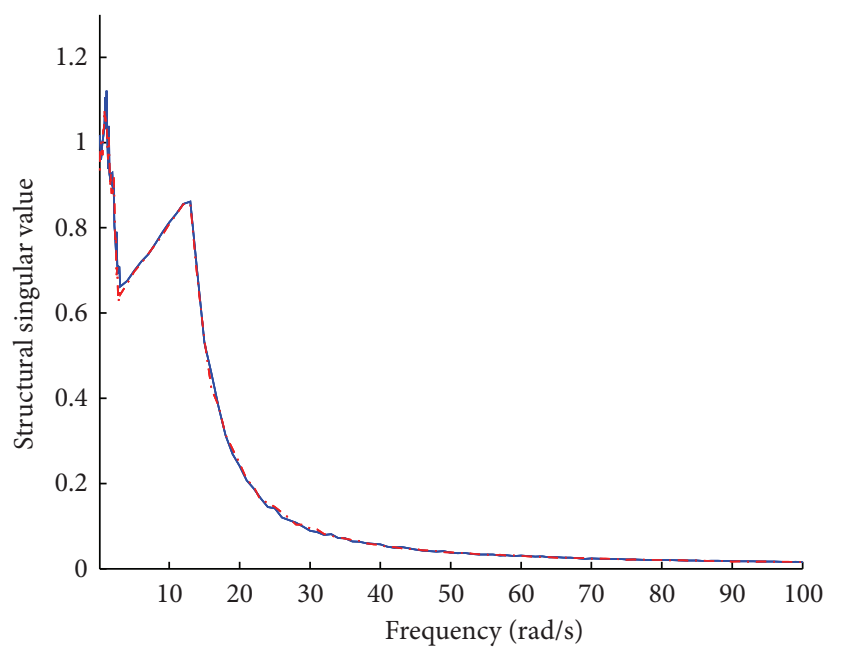

- 8 uncertainties

-.- 5 uncertainties

FIGURE 7: Structural singular value of the uncertain systems.

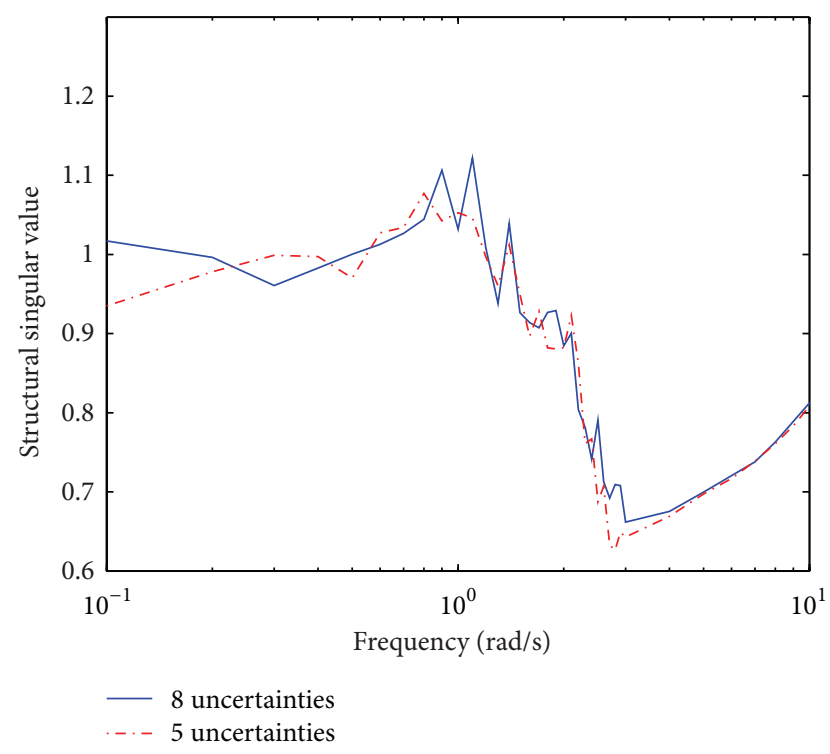

FIGURE 8: Structural singular value in low frequency interval of the uncertain systems.

is relative index. It means that we can order the effect of all uncertainties based on the GUSA index, but we cannot find a threshold value to ignore unimportant uncertainties. There is a tradeoff between precision and computational time when ignoring unimportant uncertainties. For instance, if we need to reduce more computational time, we can ignore one more uncertainty $\sigma_{6}$.

\section{Conclusion}

In this paper, a new $\mu$ framework based on GUSA is proposed. GUSA serves as the preprocessing step of $\mu$ analysis and obviously release the computational burden. Based on our

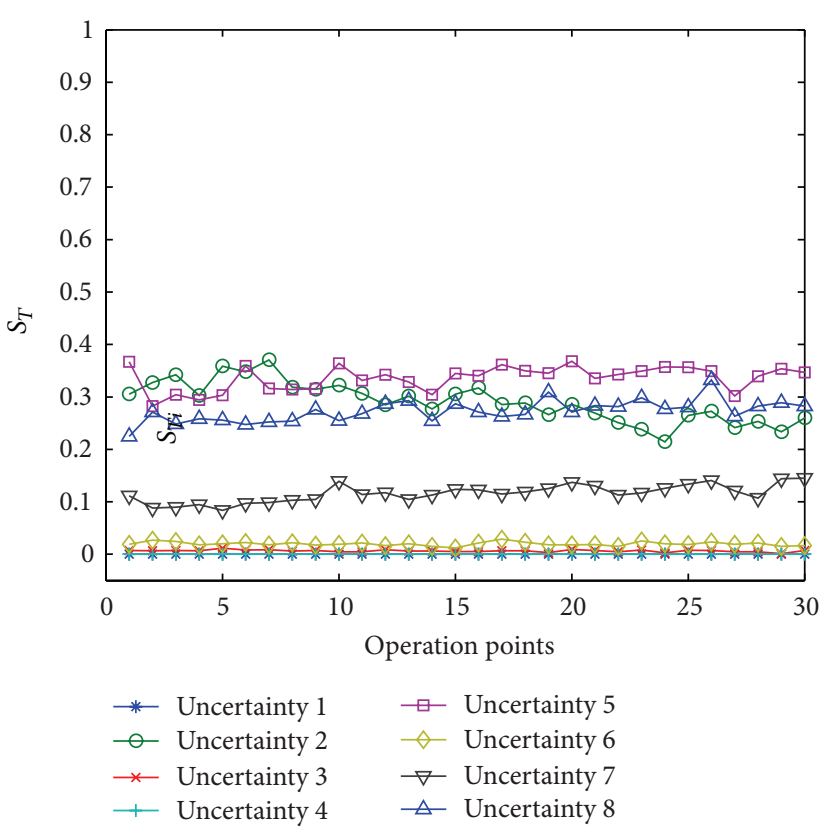

FIGURE 9: GSA of all operation points.

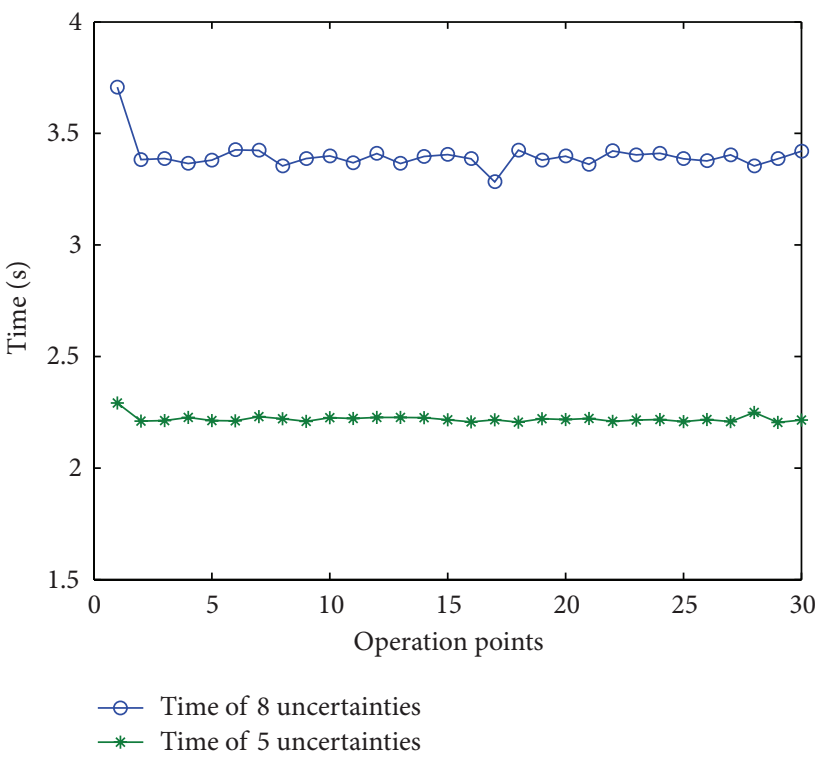

Figure 10: Computational time of $\mu$ for all operation points.

proposed stability analysis function, Sobol's method is extended and applied to determine the major uncertainties because of its maturity and flexibility. An example is given to illustrate the efficiency of the proposed method. In the potential applications, this work provides an easy-to-follow procedure for engineers to digest and expand.

\section{Conflict of Interests}

The authors declare that there is no conflict of interests regarding the publication of this paper. 


\section{Acknowledgments}

This work is supported in part by the National Nature Science Foundation of China (nos. 61203081 and 61174079), Doctoral Fund of Ministry of Education of China (no. 20120142120091), and Precision manufacturing technology and equipment for metal part (no. 2012DFG70640).

\section{References}

[1] M. Selier, U. Korte, C. Fielding, and R. Luckner, "New analysis techniques for clearance of flight control laws," in Proceedings of the AIAA Guidance, Navigation, and Control Conference and Exhibit, 14, p. 11, Austin, Tex, USA, August 2003.

[2] J. Hanson, "Advanced guidance and control project for reusable launch vehicles," in Proceedings of the AIAA Guidance, Navigation, and Control Conference and Exhibit, 2000.

[3] J. Hanson and R. Jones, "Advanced guidance and control methods for reusable launch vehicles: test results," in Proceedings of the AIAA Guidance, Navigation, and Control Conference and Exhibit, Monterey, Calif, USA, 2002.

[4] D. G. Bates, R. Kureemun, and T. Mannchen, "Improved clearance of a flight control law using $\mu$-analysis techniques," Journal of Guidance, Control, and Dynamics, vol. 26, no. 6, pp. 869-884, 2003.

[5] K. Zhou, J. C. Doyle, and K. Glover, Robust and Optimal Control, Prentice Hall, Upper Saddle River, NJ, USA, 1996.

[6] F. Corraro and M. Virgilio, "A polynomial based clearance method," in Proceedings of the AIAA Guidance, Navigation, and Control Conference and Exhibit, Austin, Tex, USA, 2003.

[7] M. H. Lowenberg and P. P. Menon, in Proceedings of the AIAA Guidance, Navigation and Control Conference and Exhibit, Hilton Head, SC, USA, 2007.

[8] P. P. Menon, J. Kim, D. G. Bates, and I. Postlethwaite, "Clearance of nonlinear flight control laws using hybrid evolutionary optimization," IEEE Transactions on Evolutionary Computation, vol. 10, no. 6, pp. 689-698, 2006.

[9] A. Yazıc1, A. Karamancıoğlu, and R. Kasimbeyli, "A nonlinear programming technique to compute a tight lower bound for the real structured singular value," Optimization and Engineering, vol. 12, no. 3, pp. 445-458, 2011.

[10] P. Seiler, A. Packard, and G. J. Balas, "A gain-based lower bound algorithm for real and mixed $\mu$ problems," Automatica, vol. 46, no. 3, pp. 493-500, 2010.

[11] L. Liu, Advanced Verification and Clearance Techniques for Modern Flight Control Systems, National Defense Industry Press, Beijing, China, 2010.

[12] S. Juliana, Q. P. Chu, and J. A. Mulder, "Reentry flight clearance using interval analysis," Journal of Guidance, Control, and Dynamics, vol. 31, no. 5, pp. 1295-1307, 2008.

[13] U. Tancredi, M. Grassi, F. Corraro, and E. Filippone, "Robustness analysis for terminal phases of reentry flight," Journal of Guidance, Control, and Dynamics, vol. 32, no. 5, pp. 1679-1683, 2009.

[14] P. P. Menon, D. G. Bates, and I. Postlethwaite, "Nonlinear robustness analysis of flight control laws for highly augmented aircraft," Control Engineering Practice, vol. 15, no. 6, pp. 655-662, 2007.

[15] W. Sun, Y. Zhao, J. Li, L. Zhang, and H. Gao, "Active suspension control with frequency band constraints and actuator input delay," IEEE Transactions on Industrial Electronics, vol. 59, no. 1, pp. 530-537, 2012.

[16] W. Sun, H. Gao, and O. Kaynak, "Adaptive backstepping control for active suspension systems with hard constraints," IEEE/ ASME Transactions on Mechatronics, vol. 18, no. 3, pp. 10721079, 2013

[17] W. Sun, Z. Zhao, and H. Gao, "Saturated adaptive robust control for active suspension systems," IEEE Transactions on Industrial Electronics, vol. 60, no. 9, pp. 3889-3896, 2013.

[18] T. Mannchen, D. G. Bates, and I. Postlethwaite, "Modeling and computing worst-case uncertainty combinations for flight control systems analysis," Journal of Guidance, Control, and Dynamics, vol. 25, no. 6, pp. 1029-1039, 2002.

[19] M. J. Hayes, D. G. Bates, and I. Postlethwaite, "New tools for computing tight bounds on the real structured singular value," Journal of Guidance, Control, and Dynamics, vol. 24, no. 6, pp. 1204-1213, 2001.

[20] C. Zhao and F. He, Multivariable Robust Control Systems, Harbin Institute of Technology Press, Harbin, China, 2010.

[21] Z. H. Zhang and Y. H. Suo, "Stability and sensitivity analysis of a plant disease model with continuous cultural control strategy," Journal of Applied Mathematics, vol. 2014, Article ID 207959, 15 pages, 2014.

[22] A. Saltelli, M. Ratto, T. Andres et al., Global Sensitivity Analysis: The Primer, John Wiley \& Sons, Chichester, UK, 2008.

[23] I. Sobol, "Sensitivity analysis for non-linear mathematical models," Mathematical Modeling \& Computational Experiment, vol. 1, pp. 407-414, 1993.

[24] J. Jacques, C. Lavergne, and N. Devictor, "Sensitivity analysis in presence of model uncertainty and correlated inputs," Reliability Engineering and System Safety, vol. 91, no. 10-11, pp. 1126-1134, 2006.

[25] A. Saltelli, P. Annoni, I. Azzini, F. Campolongo, M. Ratto, and S. Tarantola, "Variance based sensitivity analysis of model output. Design and estimator for the total sensitivity index," Computer Physics Communications, vol. 181, no. 2, pp. 259-270, 2010.

[26] J. X. Shen and W. Q. Li, "Sensitivity analysis of wavelet neural network model for short-term traffic volume prediction," Journal of Applied Mathematics, vol. 2013, 10 pages, 2013.

[27] H. Li, Guidance and Control Technology of Hypersonic Flight Vehicle, China Astronautic Publishing House, Beijing, China, 2012.

[28] Y. R. Huang, Intelligent Optimization and Its Application, National Defence Industry Press, Beijing, China, 2008. 


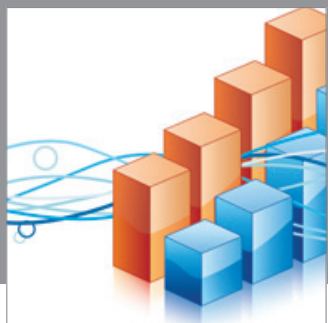

Advances in

Operations Research

mansans

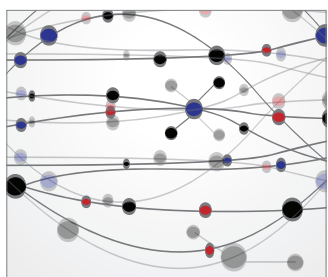

The Scientific World Journal
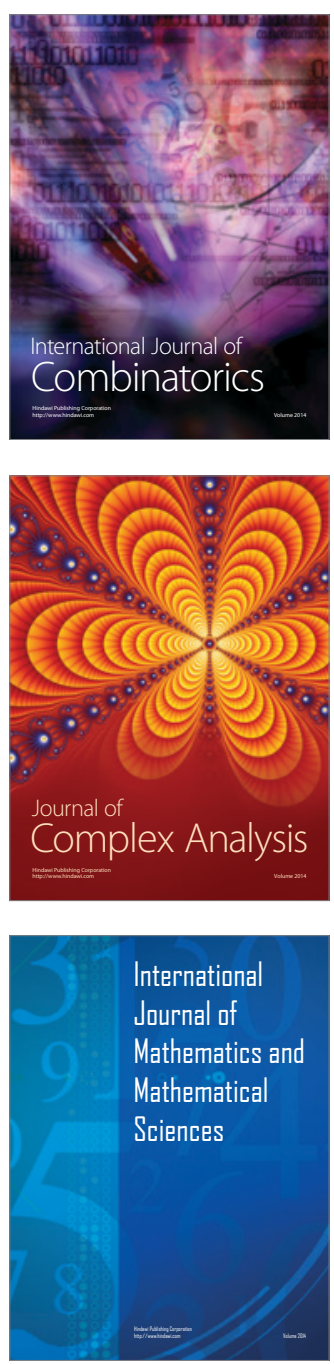
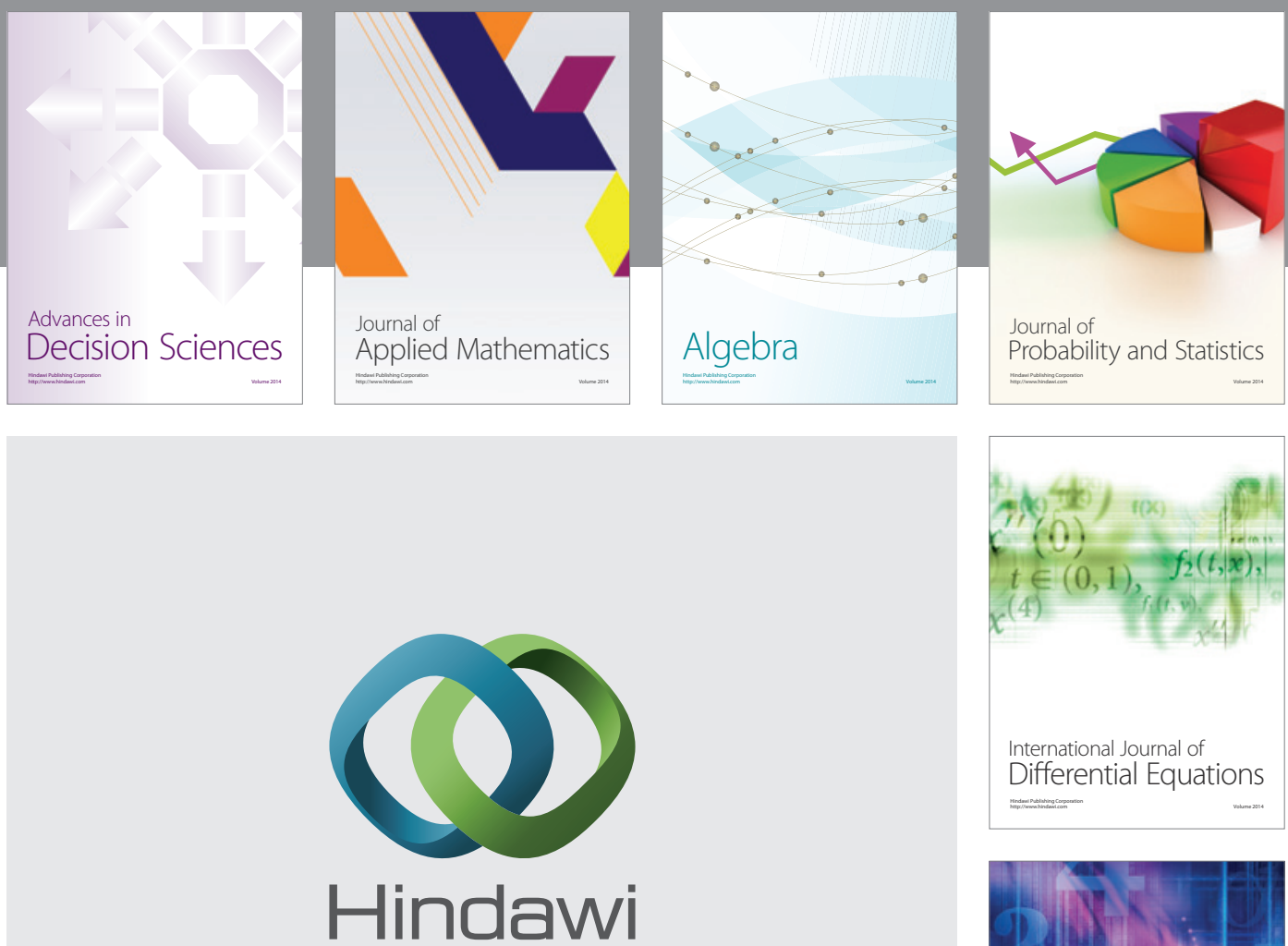

Submit your manuscripts at http://www.hindawi.com
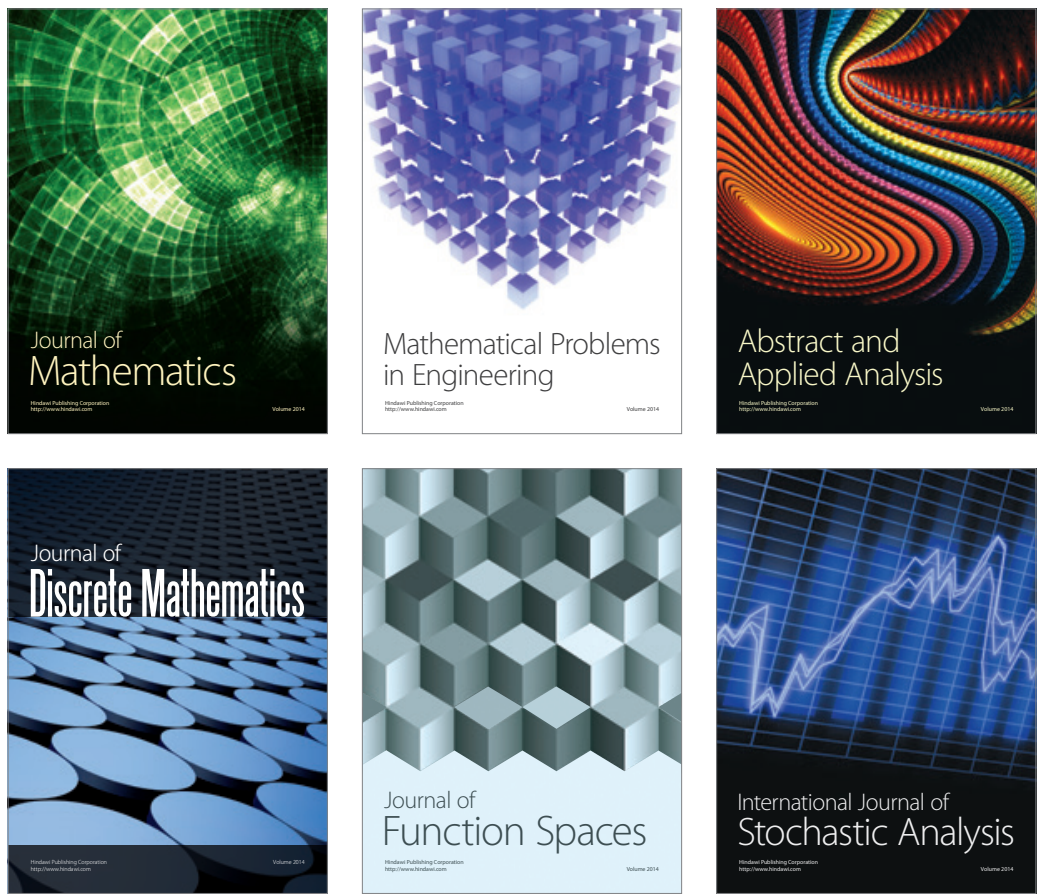

Journal of

Function Spaces

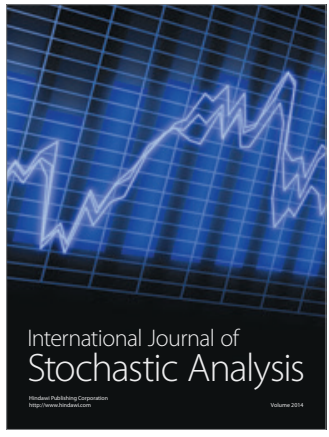

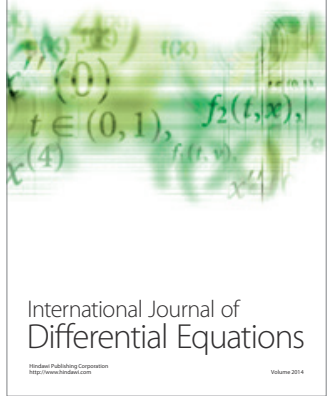
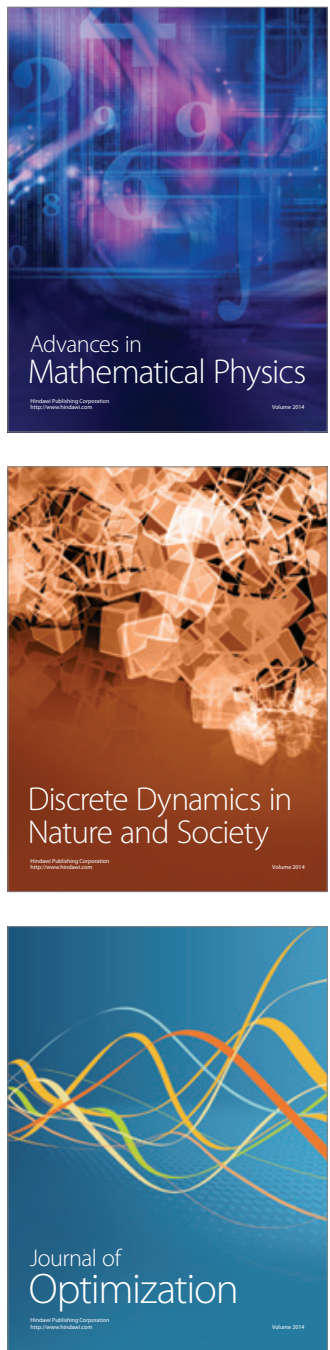